Voix et Images

voixetimages

\title{
Lectures et lecteurs
}

\section{Robert Dion}

Volume 27, numéro 2 (80), hiver 2002

La sociabilité littéraire

URI : https://id.erudit.org/iderudit/290065ar

DOI : https://doi.org/10.7202/290065ar

Aller au sommaire du numéro

\section{Éditeur(s)}

Université du Québec à Montréal

\section{ISSN}

0318-9201 (imprimé)

1705-933X (numérique)

Découvrir la revue

\section{Citer cet article}

Dion, R. (2002). Lectures et lecteurs. Voix et Images, 27(2), 365-370.

https://doi.org/10.7202/290065ar d'utilisation que vous pouvez consulter en ligne.

https://apropos.erudit.org/fr/usagers/politique-dutilisation/ 


\section{Recherche}

\section{Lectures et lecteurs}

\section{Robert Dion, Université du Québec à Rimouski}

"Qu'est-ce qu'une ceuvre classique?", se demande d'entrée de jeu Daniel Chartier dans son ouvrage intitulé L'émergence des classiques. La réception de la littérature québécoise des années $1930^{1}$. Et qu'est-ce qui fonde les traditions de lecture? Ce sont là les interrogations qui guident le travail de l'auteur dans ce qui fut d'abord une thèse de doctorat présentée à l'Université de Montréal. Pour asseoir son propos et aborder ces problèmes difficiles, Chartier a choisi les années 1930, années fertiles s'il en est, qui voient entre autres la publication des Poëmes d'Hankéou de Grandbois, de Regards et jeux dans l'espace de Saint-Denys Garneau - ouvres qui ne font pas partie du corpus, l'auteur ayant décidé de se limiter au roman et, accessoirement, à la dramaturgie - d'Un bomme et son pécbé de Claude-Henri Grignon, de Menaud, maitre-draveur de Félix-Antoine Savard, de Trente arpents, de Ringuet, des Demicivilisés de Jean-Charles Harvey pour ne nommer, cette fois, que des fictions auxquelles s'attarde l'analyste. À ces "classiques" de la littérature romanesque, s'ajoutent encore quelques textes qui auraient peutêtre pu prétendre à un tel statut, mais qui ont échoué pour diverses raisons: La chair décevante de Jovette Bernier, Dans les ombres d'Éva Senécal, ainsi que les pièces d'Yvette Ollivier Mercier-Gouin.
Un simple coup d'œil à cette liste permet de pointer une première ambiguité, qui frappe la notion même de "classique". Si Chartier s'aventure assez loin dans l'analyse des facteurs qui assurent la survie d'une ceuvre, il se préoccupe peu de définir ce qu'il entend par un classique: on doute en effet que Menaud, maître-draveur puisse être considéré comme une cuvre classique au même titre qu'Un bomme et son pécbé. Et si les deux romans ont effectivement survécu, ils n'ont pas atteint, me semble-t-il, la même stature, et ne constituent pas des classiques pour les mêmes lecteurs.

Quant au postulat initial suivant lequel les années 1930 représenteraient un "âge d'or de la critique" au cours duquel elle se serait elle-même consacrée en consacrant ses premiers classiques, il clemanderait également à être nuancé: l'uâge de la critique", dont parlait Pierre Hébert dans sa présentation du dossier éponyme publié dans Voix et images ${ }^{2}$, correspond à une période qui précède l'uâge de la parole" et non pas forcément à un âge d'or; s'il est vrai que la décennie se caractérise par un discours critique relativement nourri et diversifié, il faut voir que de nombreuses variétés de la critique, et non des moindres, étaient encore à peu près inconnues au Québec à l'époque. Encore au début des années 
1950, Roger Duhamel notait par exemple ceci :

\begin{abstract}
Il est clair qu'il n'existe pas encore ici une véritable critique universitaire. L'étude des textes, étude intrinsèque et extrinsèque, la comparaison des diverses leçons, l'analyse des variantes, la recherche approfondie des sources et des influences subies, les tentatives de littérature comparée, l'établissement définitif d'un texte, nous n'avons rien de tout cela ${ }^{3}$.
\end{abstract}

Cela dit, pourquoi insister sur l'importance de la critique durant la décennie 1930, à la fois comme genre et comme métadiscours? C'est que, pour Chartier, la critique est posée - avec raison - comme essentielle à l'accession à la qualité de classique: "La mise au jour et l'étude du système de réception à la parution, composé de l'ensemble des interventions publiques issues de la première lecture d'une œuvre, nous permettront de démontrer, dans cet ouvrage, que la survie d'une ouvre dépend de la réussite de ce passage, du bon fonctionnement de ce système." (p. 30)

Les trois premiers chapitres, sur Un bomme et son péché, Menaud, maître-draveur et Trente arpents, s'attachent à trois "réussites" critiques. La minutieuse reconstitution des débats autour de ces œuvres ${ }^{4}$ donne à Chartier l'occasion de brosser un tableau vivant, foisonnant d'anecdotes, du petit monde littéraire des années 1930. Cela ne l'empêche pas de tirer des conclusions plus générales, plus théoriques. Ainsi, le roman de Charles-Henri Grignon aurait durablement modifié l'horizon d'attente du genre romanesque dans la littérature québécoise: "[l]'habile conjonction de réalisme et de natura- lisme a aidé, affirme l'auteur, à mettre fin à un régime d'autocensure qui empêchait toute expression qui ne concordait pas directement avec l'idée de représentation nationale" (p. 108). Pour ce qui est de Menaud, maitredraveur, la facture génériquement hybride de l'ouvrage aurait forcé la critique à se faire "formaliste" ou, du moins, à accueillir les considérations formelles (p. 127). Enfin, c'est sur le plan de l'" exigence de représentation nationale et de la morale que Trente arpents se serait révélé libérateur: réussissant à racheter le caractère déceptif de son portrait de la réalité paysanne par la pureté du style (d'ailleurs reconnue par la critique française), il aurait frayé la voie à des représentations non idéalisées de la société québécoise (p. 172-173).

On ne mettra pas en cause l'intérêt de ces résultats. J'aimerais toutefois soulever une interrogation sur la vision de la critique comme accumulation de gains successifs: est-il sûr qu'une œuvre donnée puisse ouvrir une brèche dans laquelle s'engouffreront les œuvres suivantes? Les gains que décrit Chartier sont-ils nécessairement durables et irréversibles? La critique se développe-t-elle sur le socle d'une mémoire cumulative? Voilà qui aurait pu susciter de passionnantes discussions. J'ai une autre réserve importante à propos de la thèse selon laquelle "l'accumulation de ces discours [critiques] et la concurrence qui les oppose conduisent à la production d'un discours consensuel, sinon dominant" (p. 22). Les analyses tendent en effet à montrer le contraire, sauf à sauver la thèse in extremis au moyen d'un véritable glissando analytique: c'est ce qui se passe avec le roman de Grignon, 
lorsque Chartier, sans trop se justifier, assigne peu ou prou la fonction synthétique à un seul article de René Garneau. Au sujet du roman de Savard, par ailleurs, l'auteur déplore l'absence d'une synthèse "qu'il devrait y avoir eu" (p. 126); même échec du discours dominant et synthétique en ce qui a trait à La cbair décevante et à Dans les ombres (p. 186); quant aux Demi-civilisés, la désorganisation du système de réception à la suite de la censure "empêche le déroulement normal du processus critique et la production d'un discours synthétique sur l'ouvre" (p. 239, note 150 ; je souligne). Quelle est donc cette "normalité " que contredit sans cesse la réalité? L'auteur revient en conclusion sur ce supposé "discours unifié " produit par la critique et relayé par les historiens de la littérature, à qui incomberait la tâche d'adouber les classiques; mais au lieu de prendre acte des résultats de ses propres analyses, il maintient obstinément que la faillite du consensus reste l'exception (p. 283).

Les chapitres IV et VI, respectivement sur Jovette Bernier et Éva Senécal et sur Yvette Ollivier MercierGouin, ont le mérite de ramener sur le devant de la scène des écrivaines peut-être injustement oubliées. Par rapport à l'économie générale du livre, il s'agit toutefois d'un retour en arrière, à la fois chronologique (chose assez secondaire) et en quelque sorte interprétatif (c'est plus important): non seulement les œuvres des deux romancières nous replongent au tout début de la décennie, mais leur analyse fait resurgir des contraintes de réception - éthiques et morales tout spécialement - dont la première partie de l'ouvrage avait montré la progressive liquidation. Ce double recul donne ainsi l'impression que la deuxième partie du livre défait ce que la première avait érigé.

De l'ensemble du travail de Chartier, on retiendra néanmoins les nombreux aperçus éclairants sur la critique des années 1930 - qui, soit dit en passant, a été très soigneusement colligée. Au terme de la lecture, on voit mieux comment l'horizon d'attente de la littérature québécoise a été progressivement déplacé et comment la critique a peu à peu cessé de faire recommencer la littérature nationale à chaque nouvelle publication. Ce n'est pas peu.

\section{***}

C'est une tout autre vue sur la lecture que propose Lucie Hotte dans Romans de la lecture, lecture du roman. L'inscription de la lecture 5 . Visant à "combler certaines lacunes, en particulier en ce qui a trait aux médiations entre le texte, le lecteur et la lecture" (p. 29), sa réflexion prend appui sur un certain nombre d'œuvres québécoises mettant en scène des personnages de lecteurs, notamment Au pied de la pente douce de Roger Lemelin, Trou de mémoire d'Hubert Aquin, Le libraire de Gérard Bessette, Le nez qui voque de Réjean Ducharme, L'ogre de Grand Remous de Robert Lalonde et L'écureuil noir de Daniel Poliquin. L'analyse se trouve donc en quelque sorte à prolonger, sur un autre versant, les travaux aujourd'hui classiques d'André Belleau sur l'écrivain fictif, de même que quelques entreprises plus récentes. Mais l'accent ne se porte pas d'abord sur l'étude des ouvres; 
Hotte insiste surtout sur l'aspect théorique de sa démarche, qui cherche à "concilier [...] deux positions théoriques, le plus souvent perçues comme incompatibles, soit celle qui ne voit dans la lecture qu'un effet de texte et celle qui soutient que l'acte de lecture est irréductible à sa représentation textuelle ou encore à la structuration du texte" (p. 34). On reconnaîtra ici un thème de réflexion qui, ces dernières années, a occupé des universitaires québécois tels que Gilles Thérien, Bertrand Gervais et Richard Saint-Gelais, au reste régulièrement interpellés au cours de la démonstration.

Après l'obligatoire mise en situation théorique, lauteure indique le plan - un tantinet alambiqué qu'elle suivra dans l'ouvrage: une première partie qui s'attachera à dégager les diverses fonctions de la représentation de la lecture, les modes de lecture et dinterprétation des personnages lecteurs, les types de liens intertextuels entre lectures de fiction, les formes de mises en abyme de la réception; une seconde section consacrée à l'étude de romans "qui présentent des lectures en acte, c'est-à-dire dans lesquels un personnage ou un narrateur font état, souvent de façon clétaillée, de leur lecture et de leur processus d'interprétation" (p. 36).

La première fonction de la lecture que retient Hotte est la fonction référentielle, qui renvoie à la caractérisation des personnages (tous ne lisent évidemment pas la même chose), caractérisation aussi bien sociale qu'intellectuelle ou idéologique; les lectures des protagonistes ont un sens, souligne l'auteure, et celui-ci est institutionnel, ou plutôt "institutionnalisé ". La deuxième fonction est la fonction intertextuelle; Hotte se demande ici "à quelles conditions l'intertextualité devient [...] un mode d'inscription de la lecture" (p. 72). Il appert que les lectures de textes existants par les protagonistes de romans permettent au lecteur réel - par exemple à celui qui, ayant lu les Contes de Perrault, parcourt L'ogre de Grand Remous - d'inférer la suite de l'histoire. L'intertextualité peut également jouer sur le plan générique (par exemple: l'utilisation de structures issues de romans policiers dans des romans non policiers); dans ce cas aussi, sa fonction est inférentielle. La troisième fonction est décrite par Hotte comme la fonction autoréférentielle, qui concerne autant "la présence de lectures intratextuelles qui pourraient refléter la lecture extratextuelle" (p. 85) que la mise en abyme de la réception du récit.

On arrive ensuite à la cleuxième partie de l'ouvrage. Les lecteurs en acte étudiés dans cette section du livre sont envisagés dans leur dimension transgressive: pour Hotte, c'est en effet dans la mesure où les lecteurs personnages remettent en cause des conventions de lecture qu'ils accèdent à la métalecture (p. 114). Cette position me paraît plus ou moins solide: après tout, la scolastique, avec ses règles explicites, et les autres formes de lectures normées ne déploient-elles pas tout un savoir métalectural, y compris sur les lectures "déviantes" qu'elles s'acharnent à combattre? Les premières conventions que passe en revue l'auteure sont celles du genre; Le nez qui voque sert ici d'illustration, parce qu'il "met en ceuvre cette double détermination générique de la lecture, pré- 
textuelle et textuelle, en opposant son inscription générique paratextuelle ["roman", en l'occurrence] (ellemême assez ambiguë en soi) à ses marqueurs textuels" (p. 123). Les "marqueurs textuels", dans Le nez qui voque, sont constitués par les nombreuses allusions génériques au moyen desquelles le texte questionne son affiliation générique, interrogeant du même coup le rôle de la catégorie du genre dans la structuration de la lecture (p. 131). Trou de mémoire s'attaque de son côté aux conventions narratives; les dispositifs complexes dont use Aquin pour brouiller la narration et rendre à peu près impossible l'interprétation univoque rendent particulièrement visible le rôle des personnages lecteurs dans la configuration de la lecture réelle. Le roman, en effet, est construit de façon à ce que "le lecteur (externe) ne li[sel pas le texte directement, mais qu'il li[se] avant tout une lecture qui précède la sienne et qui s'impose comme essentielle à sa compréhension " (p. 147); incohérente, contradictoire, cette lecture interne lui est, au bout du compte, d'un piètre secours, et le personnage lecteur a beau se situer dans le champ de vision du lecteur réel, l'obligeant par là à revoir sa propre position de lecture, il n'offre pas pour autant de solution plausible à sa perplexité.

La conclusion de l'ouvrage est le prétexte, pour l'auteure, à revenir sur les questions théoriques formulees en introduction et à envisager, entre autres, les rôles respectifs du texte et du lecteur dans la lecture ${ }^{7}$. On pourra trouver ses conclusions un peu rapides, un peu trop simples, compte tenu de la sinuosité et de la subtilité du parcours qu'elle nous a fait suivre auparavant. Mais peut-être est-ce le destin de toutes les conclusions que d'être un peu décevantes... N'empêche que l'ouvrage est au total fort intéressant, en particulier en raison de sa construction - certes complexe, mais justifiée par l'articulation rigoureuse des différentes sousproblématiques - et de l'imbrication de la théorie et cle l'analyse: ce ne sont pas les parties qui font surtout la valeur de ce travail, c'est plutôt la somme de celles-ci.

\section{**}

Le premier livre de Yannick Roy, nous dit le bandeau publicitaire, est un "Essai sur les mauvais lecteurs dans le roman. Ce n'est pas mentir sur la marchandise, c'est bien ce que nous offre La caverne de Montésinos $^{8}$, qui s'attache aux cas de Don Quichotte et d'Emma Bovary, dont la réputation de médiocres lecteurs n'est plus à faire (comme le rappelait il y a quelques années Philippe Hamon, Emma manifeste une dangereuse propension à prendre les signes pour des consignes...). Je ne m'arrêterai pas longuement sur cet ouvrage qui ne s'inscrit pas dans le domaine des études québécoises, sinon pour compléter mon compte rendu de quelques travaux récents, publiés au Québec, portant sur divers aspects de la lecture.

Bien que Roy revendique pour son livre la désignation générique "essai ", sa démarche n'est pas étrangère aux recherches des philosophes du langage sur le statut de la fiction. De manière tout à fait parallèle à celle de Searle, de Pavel, de Ryan ou de Hamburger, Roy est amené à croi- 
ser sans cesse le problème de la véracité des assertions en domaine fictionnel, de l'interprétation des actes de langage indirects tels que l'ironie, du statut logique de la feintise et des univers de fiction. Pour lui, la fiction est le domaine de la croyance ironique, et les ceuvres de Cervantès et de Flaubert le montrent à l'envi. Or Don Quichotte et $\mathrm{Ma}$ dame Bovary ne remettent pas seulement en cause la lecture des personnages de romans; le lecteur réel est lui aussi contaminé par ces mauvaises lectures dont il est forcé de prendre connaissance pour entrer dans le monde de la fiction. Car si Don Quichotte et Emma semblent nier la "réalité" dans laquelle ils vivent et se situer de plain-pied dans un monde de fiction, comment le lecteur réel pourrait-il à son tour être sûr de la réalité du monde fictif à l'intérieur duquel ils se trouvent? N'est-il pas placé au confluent de deux univers fictifs qui se contredisent? Par une suite de raisonnements serrés, Roy en arrive à la thèse suivante, qui est celle de tout l'ouvrage:

Le mauvais lecteur n'est donc
mauvais que s'il est une personne
réelle, c'est-à-dire s'il offre au
regard d'une personne qui
occupe le même "étage" ontolo-
gique que lui. À partir du moment
où il devient fictif et s'offre au
regard d'un lecteur réel, il est en
quelque sorte sauvé, puisque la
réalité à laquelle il s'oppose par
son statut de mauvais lecteur est
elle-même invalidée, fragilisée, du
seul fait qu'elle est une réalité fic-
tive. (p. 56)

Dans un monde fictif où la soi-disant réalité est aussi précaire que les chimères de mauvais lecteurs, dans des romans dont les auteurs refusent de nous donner la clef, pratiquant une ironie pour ainsi dire généralisée, le lecteur réel ne disposerait en définitive d'aucun point d'appui pour décider du vrai et du faux, du sérieux et du fictif...

Bref, voici un petit essai stimulant, ludique - c'est là le vou de l'auteur - et en même temps sérieux (comme la fiction?), en prise sur des questions actuellement largement débattues, et qui débouche sur des aperçus éclairants à propos des savoirs de la littérature.

1. Daniel Chartier, L'émergence des classiques. La réception de la littérature québécoise des années 1930, Montréal, Fides, coll. "Nouvelles Études québécoises", 2000.

2. Dossier "L'Âge de la critıque, 1920-1940", Voix et images, $\mathrm{n}^{\circ}$ 50, hiver 1992.

3. Roger Duhamel, "La critıque et le critıque", La Nonvelle Reune canadienne, vol. $1, \mathrm{n}^{\circ} 2$, 1951, p. 23-34.

4. En fait, cette reconstitution est beaucoup plus détaıllée dans le premier chapitre sur Un homme et son péché. Chartier y retrace en effet le discours critique sur tous les avatars du roman de Grignon adaptations radiophonique et théâtrale, film, série télévisée. En comparaison, le chapitre sur Menalld, maître-draveur apparaît pour le moins sommaire, qui ne fait pas même écho aux diverses réécritures de l'œuvre par Savard.

5. Lucie Hotte, Romans de la lecture, lecture du roman. L'inscription de la lecture, Québec, Éditions Nota bene, coll. "Littérature(s)", 2001.

6. Je pense entre autres à la thèse de Roseline Tremblay, Le poète et le porte-parole. Sociogramme de lécrivain dans le roman québécois (1960-1995) (UQAM/Université de Paris-VIII, 1999), et à mes propres travaux (Le moment critique de la fiction).

7. "Le rôle du texte dans l'élaboration de la lecture consiste à inscrire un parcours de lecture; le rôle du lecteur consiste à décider du choix des lectures, du but poursuivi et du mode de lecture." (p. 151)

8. Yannick Roy, La caverne de Montésinos, Québec, Éditions Nota bene, 2001. 